\title{
Mehr gesunde Babys
}

\author{
More Healthy Babies
}

G. Jorch, D. Körholz, L. Gortner

Bibliografie

DOI http://dx.doi.org/

$10.1055 / \mathrm{s}-0033-1358724$

Klin Padiatr 2013; 225: 377-378

(c) Georg Thieme Verlag KG

Stuttgart $\cdot$ New York

ISSN 0300-8630

Korrespondenzadresse

Prof. Dr. Gerhard Jorch Universitätskinderklinik Leipziger Straße 44 39120 Magdeburg gerhard.jorch@med.ovgu.de
So lautet der Slogan einer Aktion deutscher medizinischer Fachgesellschaften und Elternverbände, die Ende 2011 begonnen wurde [9]. Es handelt sich dabei um die Propagierung eines Gesundheitszieles, welches im Wesentlichen durch die freiwillige und kreative Mitarbeit von Pränatalmedizinern, Geburtshelfern, Neonatologen und Neuropädiatern erreicht werden soll. Natürlich besteht die Erwartung auf flankierende Maßnahmen der Gesundheitspolitik, nichtärztlicher Angehöriger von medizinischen Berufen und natürlich der Eltern.

\section{Wie misst man „Gesunde Babys"?}

In entwickelten Ländern werden heute relevante Gesundheitsstörungen in der frühen Kindheit im Wesentlichen durch Frühgeburtlichkeit, angeborene Fehlbildungen und andere Störungen der Perinatalperiode verursacht [8]. Es ist evident, dass die Anzahl von Todesfällen als der schwersten Form einer Gesundheitsstörung wie die Spitze eines Eisberges einen Hinweis auf das Gesamtausmaß der Gesundheitsstörungen mit Sterberisiko und dem Risiko einer lebenslangen Behinderung gibt. Nosokomiale Infektionen sind ein gutes Beispiel dafür [3,4]. Todesfälle können zuverlässig populationsbezogen erfasst werden, jedenfalls besser als andere Gesundheitsstörungen. Dabei bleibt die Wahl zwischen Totgeborenen, neonatal bis zum 28. Lebenstag und postneonatal zwischen dem 29. und 365. Lebenstag verstorbenen Lebendgeborenen. Für die Beurteilung der durch Perinatalzentren zu beeinflussenden Ergebnisqualität ist die neonatale Mortalität, d.h. die Zahl der in den ersten Lebenstagen (frühneonatal) und vom 29.-365. Lebenstag (spätneonatal) verstorbenen Lebendgeborenen dividiert durch die Gesamtzahl der Lebendgeborenen, am besten geeignet, weil vor und nach dieser Zeitspanne wichtige Faktoren, die der Beeinflussbarkeit durch die perinatale Medizin entzogen sind, die Rate der Totgeborenen und der postneonatal verstorben Säuglinge maßgeblich bestimmen.

\section{Neonatale Mortalität - international}

Derzeit ist die neonatale Mortalität in Japan, Island und einigen Klein- und Stadtstaaten wie Singapur und Monaco mit 1 Todesfall auf 1000 Lebendgeborene am niedrigsten [7]. Unter den nachfolgenden Ländern mit 2 auf 1000 liegen u.a. Schweden, Finnland, Österreich und Deutschland $[2,7]$. Es besteht eine Korrelation zwischen der neonatalen Mortalität und dem soziökonomischen Entwicklungsstand eines Landes. Es gibt aber Ausnahmen: So ist die neonatale Mortalität in den medizinisch hochgerüsteten USA mit 4 auf 1000 höher als in Kuba mit 3 auf 1000 [7]. Es entscheiden wohl nicht nur der technologische Standard, sondern auch unbekannte „weiche Faktoren“.

\section{... und in Deutschland}

Innerhalb Deutschlands fallen bei vergleichbarem soziökonomischem Standard und ärztlichem Ausbildungsniveau erhebliche Unterschiede zwischen den Bundesländern mit 1,4 auf 1000 in Sachsen und 3,6 auf 1000 in Bremen im Zeitraum 2010-2012 auf [8]. Erfreulich daran ist, dass angesichts der auch im weltweiten Vergleich sehr niedrigen Mortalität in Mitteldeutschland, es durchaus realistisch und machbar erscheint, die gesamtdeutsche Mortalität schon in absehbarer Zeit auf japanische Werte zu senken. Wenn dies, wie aufgrund der einführenden Überlegungen zu erwarten, mit einer Senkung der Gesamtmorbidität einhergeht, sind also „mehr gesunde Babys“ als Gesundheitsziel in Reichweite.

\section{Warum sterben im Nordwesten \\ Deutschlands mehr Neugeborene als im Osten und Süden?}

Die Neugeborenensterblichkeit zeigt in den letzten Jahren einen Gradienten von Nordwest nach Südost. In den Jahren 2010-2012 war die neonatale Mortalität am höchsten in den Bundesländern, Bremen, Niedersachsen und NordrheinWestfalen und am niedrigsten in den Bundesländern Sachsen, Sachsen-Anhalt und Thüringen [8]. Dieser Gradient besteht erst seit wenigen Jahren. Er beruht nicht darauf, dass die Mortalität im Nordwesten angestiegen ist: Eine Senkung der neonatalen Mortalität ist in den letzten 10 Jahren in allen Bundesländern erzielt worden! Das Ausmaß der Senkung war aber in den mitteldeutschen Ländern am stärksten [2,8].

Über die Gründe kann man spekulieren. Jedenfalls scheint ein dichtes Netz an Perinatalzentren nicht förderlich zu sein. In den 3 derzeit führenden Bundesländern werden sehr unreife Frühgeborene, die überall den Hauptanteil der verstorbenen Neugeborenen ausmachen, im Wesentlichen nur an 6 Standorten behandelt, was zu Wegstrecken für Schwangeren mit Frühgeburtsbestrebungen von über $100 \mathrm{~km}$ führt.

Die Gesamtfläche der Bundesländer Sachsen, Thüringen und Sachsen-Anhalt beträgt $55000 \mathrm{~m}^{2}$, liegt also über der von z.B. Nordrhein-Westfalen und Baden-Württemberg mit jeweils etwa $35000 \mathrm{~m}^{2}$, die deutlich mehr Perinatalzentren mit kürzeren Abstand untereinander haben. 
Neonatalerhebung - die Weiterentwicklung eines Qualitätssicherungsinstruments

Die Qualitätssicherung in der Geburtshilfe und Neonatologie entwickelte sich historisch aus den bei den Ärztekammern angesiedelten Perinatal- und Neonatalerhebung. Hier wurde seit den 80er-Jahren des vergangenen Jahrhunderts krankenhausbezogen Ergebnisqualität (Mortalität, bestimmte Frühkomplikationen), Prozessqualität (bestimmte Untersuchungen, Körpertemperatur bei Aufnahme) und Strukturqualität (Patientenzahl) abgefragt. Methodisch waren anfangs mangelhaft die fehlende Überprüfung der Datenqualität, die unvollständige Erfassung, die dürftige Begründung der gewählten Qualitätsmerkmale und der abgeleitete Handlungsbedarf.

Durch zunehmend strukturierte Rückmeldung an die Leistungserbringer seitens der Fachkommissionen in den Projektgeschäftsstellen der Ärztekammern, die Überprüfung der erfassten Merkmale mit fachlicher Konsensbildung seitens der Bundesgeschäftsstelle Qualitätssicherung gGmbH und schließlich Beauftragung des Aqua-Instituts $\mathrm{GmbH}$ mit der bundesweiten Strukturierung und Ergebnisbewertung ab 2010 durch den gemeinsamen Bundesausschuss wurden diese Mängel weitgehend behoben bzw. sind auf dem Weg, behoben zu werden [1]. Als neueste Entwicklung wurde ein Internetportal freigeschaltet, wo - allerdings noch auf freiwilliger Basis - die Ergebnisse einzelner Perinatalzentren der Öffentlichkeit zugänglich gemacht werden.

\section{Verbesserungsvorschlag: Regionale Ergebnisqualität in den Focus rücken}

Der verbleibende Nachteil dieser demnächst optimierten Qualitätssicherungsstruktur wird sein, dass die Qualitätsmerkmale krankenhausbezogen dargestellt werden. Aus Public-HealthSicht wäre aber mindestens ergänzend eine populationsbezogene regionale Darstellung der Ergebnisqualität wünschenswert, und zwar aus folgenden Gründen:

1. Eine gute krankenhausbezogene Ergebnisqualität kann auch durch Selektion erreicht werden („Rosinen picken“).

2. Maßnahmen zur Verminderung der Frühgeburtenraten durch primäre und sekundäre Prävention führen zu einer niedrigeren neonatalen Mortalität und werden bei Fokussierung auf regionale Ergebnisqualität „belohnt“.

3. Allein krankenhausbezogene Qualitätssicherung birgt das Risiko einer Vernichtung von Ressourcen durch Konkurrenzkampf.

4. Die Fokussierung auf regionale Ergebnisqualität fördert Vernetzung und Kooperation zugunsten der Patienten.

5. Sie ist ein guter Maßstab für die regionale Krankenhausplanung.

6. Sie führt zu einer Berücksichtigung regionaler Besonderheiten bei der Planung.

Deshalb schlagen die Autoren vor, eine niedrige neonatale Mortalität auf Bundeslandebene bzw. bei größeren Bundesländern auf Regierungsbezirksebene als wichtiges Gesundheitsziel zu verfolgen. Regionen mit einer Lebendgeborenenrate von mindestens 10000 , besser 25000 sind allerdings erforderlich, um angesichts der geringen absoluten Zahl an Todesfällen statistisch hinreichend zuverlässige Zahlen zu erhalten. Für Bundesländer wie Bremen oder das Saarland mit weniger als 10000 Lebendgeborenen pro Jahr bspw. können keine zuverlässigen Angaben auf jährlicher Basis gemacht werden, sondern es müssen mehrere Jahre zusammengefasst dargestellt werden.

Weitergehende Aussagen lassen sich durch wissenschaftliche Netzwerke gewinnen, wo unter anderem auf dem Boden des German Neonatal Network (GNN) zahlreiche Studien für die
Hochrisikogruppe sehr kleiner Frühgeborener publiziert wurden. So sind die dort gegebenen Daten zur Mortalität von sehr kleinen Frühgeborenen mit Lungenversagen als wichtigste Ursache für deren Mortalität während der stationären Behandlung nachgewiesen [6]. Diese Daten helfen neben Registern, Hochrisikogruppen weiter zu identifizieren und auf dem Boden der genannten Daten Konzepte zur Verbesserung der Prognose zu erarbeiten [5].

\section{Was bringt Zertifizierung?}

Ausgehend vom Beispiel der Brustzentren-Zertifizierung gibt es Bemühungen, ähnliche Zertifizierungen für Perinatalzentren einzuführen. Man muss bei der Beurteilung dieses Weges aber wissen, dass im Wesentlichen mehr oder weniger evidenzbasierte Merkmale der Strukturqualität abgefragt werden, teilweise auch der Prozessqualität. Die aus Sicht der Patienten letztlich entscheidende Ergebnisqualität bleibt weitestgehend außen vor.

\section{Wie wichtig sind Mindestmengen?}

Das gilt im Übrigen auch für das Mindestmengenkonzept. Natürlich spricht der Augenschein dafür, dass die Voraussetzungen für eine gute Ergebnisqualität bei hinreichend großen Strukturen besser sind. Bestimmte Strukturen kann sich eine Einheit mit geringen Einnahmen wegen unzureichender Patientenzahlen nicht leisten. Auch die Behandlungskontinuität ist stärker gefährdet, wenn sie auf nur wenigen Schultern ruht. Dennoch gibt es Beispiele von unterdurchschnittlicher Ergebnisqualität trotz hoher Patientenzahlen und guter Ergebnisqualität trotz kleiner Patientenzahlen. Die Orientierung auf die Überschreitung von Mindestmengen konterkariert die Hauptaufgabe der perinatalen Medizin, sehr unreife Frühgeburten zu vermeiden.

Die erstrebenswerte Reduzierung der Zahl der Perinatalzentren muss auf anderem Weg, am besten durch Fokussierung auf Ergebnisqualität erreicht werden.

\section{Fazit}

Wenn durch die Betonung der regionalen Ergebnisqualität die in den Regionen tätigen Leistungserbringer einen Vorteil in der gestuften Vernetzung sehen, werden sie diese suchen, das potenziell Ressourcen vernichtende Konkurrenzverhalten aufgeben und nachhaltig das Ziel „Mehr gesunde Babys“ verfolgen.

\section{Literatur}

1 Bundesministerium für Gesundheit. Bekanntmachung eines Beschlusses des Gemeinsamen Bundesausschusses über eine Änderung der Anlage der Vereinbarung zur Qualitätssicherung: Leistungsbereiche 2010. BAnz. Nr. 137 (S. 3265) vom 15.09.2009

2 Gaber E. Sterblichkeit und regionale Unterschiede - Heft 52. Gesundheitsberichterstattung Robert Koch-Institut, Berlin: 2011

3 Gortner L. Nosocomial infections in very preterm neonates - improvements by further scientific research or discussions in talk shows? Klin Padiatr 2013; 225: 55-56

4 Leistner $R$. Nosokomial infections in very low birth weight infants in Germany: current data from the National Surveillance System NEOKISS. Klin Padiatr 2013; 225: 75-80

5 Stein A, Keller M, Ross S et al. Pandemic A/H1N1 (2009) influenza infections in very-low-birth-weight infants - a case series from the German Neonatal Network. Klin Padiatr 2013; 223: 267-270

6 Stichtenoth G, Demmert M, Bohnhorst B et al. Major contributors to hospital mortality in very-low-birth-weight infants: data of the birth year 2010 cohort of the German Neonatal Network. Klin Padiatr 2012; 224: $276-281$

7 www.data.worldbank.org

8 www.gbe-bund.de

9 www.rund-ums-baby.de/mehr-gesunde-babys 